\title{
Pollution des eaux à usages domestiques par les éléments traces métalliques des activités anthropiques: cas du sous bassin versant du fleuve Sassandra en amont du barrage de Buyo, Côte d'Ivoire
}

\author{
Kouakou Séraphin Konan ${ }^{1}$, Kouakou Benoit Kouamé ${ }^{1}$, Félix Koffi Konan ${ }^{2, \dagger}$, Koffi Charles Boussou ${ }^{2}$, \\ and Kouassi Lazare Kouakou ${ }^{1}$ \\ ${ }^{1}$ Laboratoire des Sciences et Technologies de l'Environnement, Université Jean Lorougnon Guédé, \\ Daloa, Côte d'Ivoire \\ ${ }^{2}$ Laboratoire de Biodiversité et Gestion Durable des Ecosystèmes Tropicaux, Université Jean Lorougnon \\ Guédé, Daloa, Côte d'Ivoire$$
\mathbf{t}_{\text {deceased }}
$$

Correspondence: Kouakou Séraphin Konan (konandks@yahoo.fr)

Published: 16 November 2021

Résumé. Pour résoudre les problèmes d'approvisionnement en eau potable des centres urbains, le gouvernement ivoirien a décidé d'utiliser les ressources pérennes telles que le fleuve Sassandra. Ainsi, le sous bassin du fleuve Sassandra en amont du barrage de Buyo est pressenti pour alimenter les villes de la région du Haut Sassandra et du département de Duekoué. Cependant, les intrants agricoles, les activités d'orpaillage et les déchets ménagers entrainent une forte contamination des eaux de ce fleuve en éléments traces métalliques (ETM). Ainsi, les tendances spatiales et temporelles de la pollution métallique ont été évaluées par le calcul des facteurs d'enrichissement, des indices de géo-accumulation, de l'indice de charge polluante, de l'indice de pollution sédimentaire et de l'indice de risque écologique individuel sur vingt-huit (28) échantillons provenant de sédiments de surface de ce fleuve. Les résultats montrent que des pollutions modérées à modérément sévères sont enregistrées au niveau du mercure $(\mathrm{Hg})$ et du cuivre $(\mathrm{Cu})$. Le $\mathrm{Pb}$, l'As et le $\mathrm{Cd}$ connaissent un enrichissement modéré, avec des facteurs d'enrichissement respectifs de 1,77; 1,60 et 1,79 suggérant l'existence d'autres sources de métaux autres que la source naturelle terrigène. L'indice de pollution sédimentaire estimé à 5,95 $(<10)$ montre que les sédiments sont moyennement pollués. Le risque écologique moyen lié aux $\mathrm{Cd}, \mathrm{As}, \mathrm{Pb}, \mathrm{Cu}$ et $\mathrm{Zn}$ est largement inférieur à 40 traduisant un risque bas en référence à ces métaux avec le risque écologique moyen lié au $\mathrm{Hg}$ (160) largement supérieur à 40. L'analyse temporelle couplée au test SEQ-Eau montre également que le stockage des éléments traces métalliques au niveau des sédiments n'est pas définitif. Les risques de relargage, de remobilisation, de biodisponibilité et donc de toxicité, sont à craindre et constituent ainsi un danger permanent pour toute la chaîne trophique. Des mesures de prévention doivent donc être prises pour anticiper sur les difficultés que pourrait rencontrer le traitement de ces eaux pour les usages domestiques. 


\section{Introduction}

Les métaux lourds sont de nature persistants, toxiques et ont une très forte tendance à s'accumuler dans les organismes vivants et à s'adsorber sur les particules de sédiment (Wang et al., 2012). La pollution par les métaux des écosystèmes aquatiques augmente en raison des effets de l'urbanisation, de l'agriculture, des activités d'orpaillage et dans une moindre mesure de l'industrialisation dans notre zone d'étude. Le sous bassin versant du fleuve Sassandra (lac Guessabo) est susceptible d'être fortement contaminé par le $\mathrm{Hg}$, le $\mathrm{Pb}$, le $\mathrm{Cd}$, le $\mathrm{As}$, le $\mathrm{Cu}$, le $\mathrm{Zn}$, le $\mathrm{Mn}$ et le $\mathrm{Fe}$ en raison du rejet croissant de polluants dans le bassin par les eaux usées des stations-services, du ruissellement des pesticides et des engrais agricoles. Les sédiments sont connus pour être des réservoirs ultimes des métaux lourds rejetés dans l'environnement (Malferrari et al., 2009). Ainsi, les sédiments pourraient constituer une source potentielle de métaux lourds qui seront rejetés dans les eaux sus-jacentes par des moyens naturels et des processus anthropiques (Beutel et al., 2008), où ils pourraient avoir des effets néfastes sur la qualité de l'eau de boisson et la santé humaine. Certains métaux toxiques dans les sédiments peuvent être mobilisés par des procédés biochimiques tels que la prolifération de cyanobactéries, causant une pollution de l'eau (Olivares-Rieumont et al., 2009). D'autres métaux lourds sont nécessaires aux organismes mais peuvent être toxiques si leurs concentrations sont assez élevées. Selon plusieurs auteurs, plus de $90 \%$ des teneurs en métaux lourds sont liées aux particules en suspension dans les hydrosystèmes soulignant ainsi l'importance de cette matrice dans l'étude de la qualité des systèmes aquatique (Wang et al., 2012). Ainsi, l'étude des sédiments permet de connaitre les changements environnementaux survenus dans les bassins fluviaux et fournissent des informations utiles sur l'accumulation de métaux lourds, reflétant les impacts naturels (géogéniques) et anthropiques. La surveillance de la concentration de métaux lourds et l'évaluation de leur niveau de contamination dans les sédiments du lac Guessabo sont des questions impératives et cruciales pour assurer la durabilité des écosystèmes. L'objectif de ce travail est de déterminer la distribution de certains métaux lourds $(\mathrm{Hg}, \mathrm{As}, \mathrm{Cd}$, $\mathrm{Pb}, \mathrm{Cu}, \mathrm{Zn}, \mathrm{Mn}$ et $\mathrm{Fe}$ ) dans le sédiment et d'évaluer le degré de contamination afin d'aider à la prise de décision pour anticiper sur les difficultés que pourrait rencontrer le traitement de ces eaux pour les usages domestiques.

\section{Matériel et Méthodes}

\subsection{Milieux d'étude}

Le lac Guessabo est situé entre les latitudes $6^{\circ} 57^{\prime}$ et $7^{\circ} 2^{\prime} \mathrm{N}$ et les longitudes $6^{\circ} 45^{\prime}$ et $6^{\circ} 46^{\prime} \mathrm{W}$ (Fig. 1). Il se trouve en amont du barrage de Buyo construit sur le fleuve Sassandra. Il couvre une superficie de $27 \mathrm{~km}^{2}$. L'agriculture constitue l'activité économique principale (Kouamé et al., 2019a). Le lac Guessabo est traversé par la nationale 6. Les activités économiques liées au milieu lacustre sont constituées essentiellement de la pêche.

\section{2 Échantillonnage}

L'échantillonnage a été fait à l'aide d'une benne Van Veen, qui a permis de prélever les 5 premiers centimètres de la colonne sédimentaire. Ces premiers centimètres correspondent à la zone la plus active (mobilité verticale et latérale, interface eau-sédiment). Les concentrations en ETMs sont parfois très faibles, c'est pour pourquoi nous avons redoublé de vigilance au moment des prélèvements et éviter toute source de pollutions externes. Il a été impératif d'utiliser des instruments en plastique décontaminés au préalable. Les échantillons recueillis ont été placés directement dans des sacs en polyéthylène scellés à l'air et conservés à $4{ }^{\circ} \mathrm{C}$ jusqu'à l'analyse. Au laboratoire, les échantillons de sédiments lacustres ont été soumis à un traitement mécanique pour obtenir une poudre dont les particules ont un diamètre inférieur à $63 \mu \mathrm{m}$.

\subsection{Analyse}

Avant l'analyse au spectrophotomètre d'absorption atomique, les échantillons prétraités de sédiments $(0,1 \mathrm{~g})$ sont minéralisés à chaud dans des tubes en téflon à l'aide de $3 \mathrm{~mL}$ d'acide hydrofluoridrique (HF) concentré pur $(48 \%)$ avec $1 \mathrm{~mL}$ d'eau régale $\left(\mathrm{HNO}_{3}+\mathrm{HCl} ; 1 / 3 v / v\right)$. L'utilisation de HF est essentielle parce qu'il est le seul acide qui dissout complètement les treillis silicatés et libère tous les métaux. Après la digestion, les résidus sont récupérés avec une solution d'acide borique (2,7 g dans $20 \mathrm{~mL}$ d'eau bidistillée). Le volume final est ramené à $50 \mathrm{~mL}$. Ce volume final est laissé au repos pendant un jour pour s'assurer de la digestion totale des échantillons. La méthode d'analyse correspond à celle décrit par Kouamé et al. (2019b).

\subsection{Contamination assessment}

Pour évaluer le niveau de contamination des sédiments par les métaux lourds, celui-ci a été évalué par le calcul de facteurs d'enrichissement, d'indices de géoaccumulation, de l'indice de charge polluante, de l'indice de pollution sédimentaire et de l'indices de risque écologique. La haute croûte continentale faisant référence à des formations sédimentaires (Wedepohl, 1995), est la référence utilisée dans ce présent travail. Aussi dans ce travail, le fer (Fe) a été choisi comme élément normalisant parce qu'il est associé à des surfaces solides fines, sa géochimie est similaire à celle de nombreux métaux traces; et sa concentration dans le sédiment naturel tend à être uniforme (Daskalakis and O'Connor, 1995). 


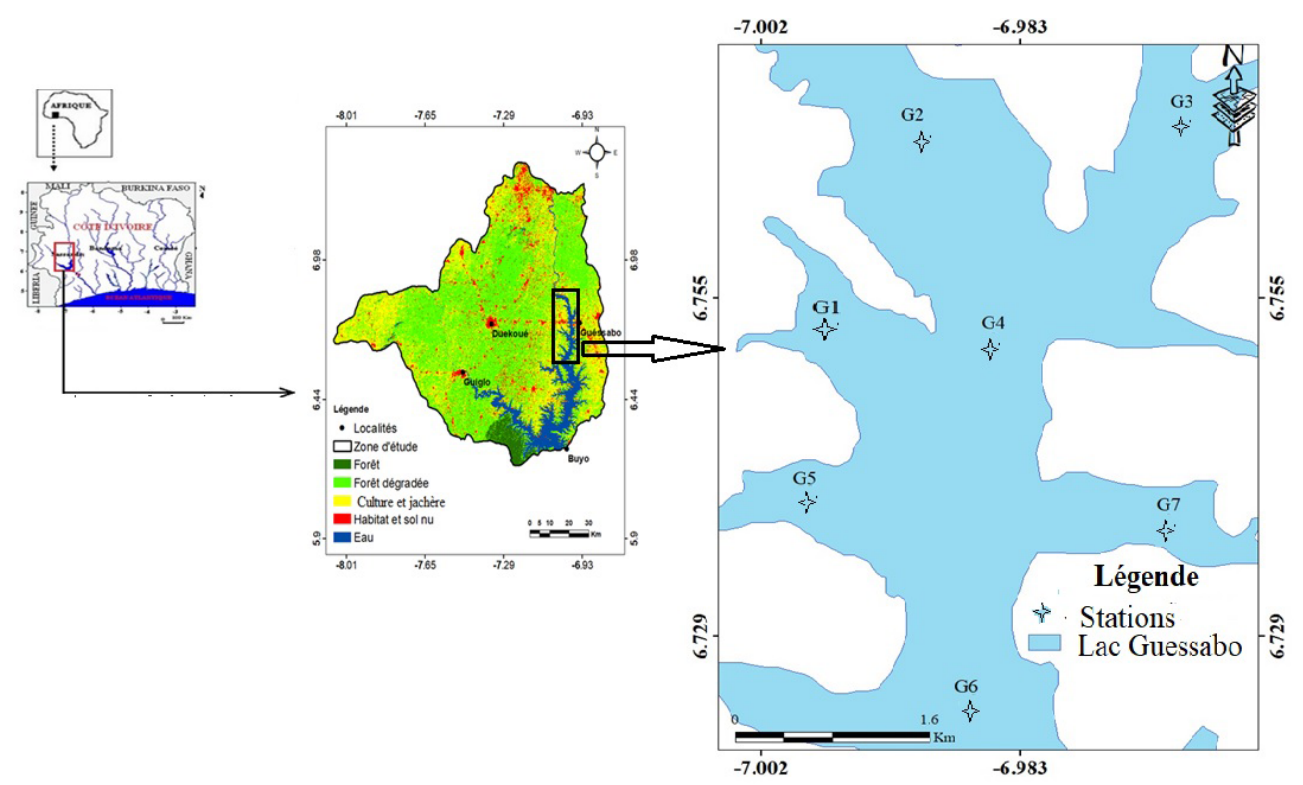

Figure 1. Stations d'échantillonnage et localisation de la zone d'étude.

\subsection{Indice de géoaccumulation}

Pour quantifier l'intensité de la pollution métallique l'index de géoaccumulation introduit par Müller (1981) est utilisé et définit par l'équation 1.

Igeo $=\log _{2}\left(\frac{C_{\mathrm{n}}}{1,5 \times B_{\mathrm{n}}}\right)$

- Igéo : indice de géo accumulation;

- Cn : concentration mesurée dans l'échantillon

- $\mathrm{Bn}$ : fond géochimique;

- 1,5: facteur d'exagération du fond

Igéo $<0$ Non contaminé; $0<$ Igéo $<1$ Non contaminé à modérément contaminé; $1<$ Igéo $<2$ Modérément contaminé; $2<$ Igéo $<3$ Modérément contaminé à sévèrement contaminé; $3<$ Igéo $<4$ Sévèrement contaminé; $4<$ Igéo $<5$ Sévèrement à extrêmement contaminé; $5<$ Igéo Extrêmement contaminé.

\subsubsection{Facteur d'enrichissement}

Le FE a été calculé pour déterminer l'origine anthropique ou naturelle des éléments dans les sédiments du lac Guessabo. Le facteur d'enrichissement fourni le nombre de fois qu'un élément est enrichi par rapport à l'abondance de cet élément dans le matériau de référence. FE est défini par l'équation 2

$\mathrm{FE}=\frac{([X] /[R])_{\text {échantillon }}}{([X] /[R])_{\text {matériel de reférence }}}$
- $R$ : concentration de l'élément normalisant dans le sédiment $\left(\mu \mathrm{g} \mathrm{g}^{-1}\right)$

- $[X]=$ concentration de l'élément trace métallique dans le sédiment $\left(\mu \mathrm{g} \mathrm{g}^{-1}\right)$

- FE $\leq 1$ Aucun enrichissement; $1<\mathrm{FE} \leq 3$ Enrichissement mineur; $3<\mathrm{FE} \leq 5$ Enrichissement modéré

$-5<\mathrm{FE} \leq 10$ Enrichissement modérément sévère, $10<$ $\mathrm{FE} \leq 25$ Enrichissement sévère; $25<\mathrm{FE} \leq 50$ Enrichissement très sévère

\subsubsection{Indice de pollution sédimentaire}

Pour l'évaluation globale de la qualité des sédiments tenant en compte de la toxicité relative de chaque métal en lui affectant un facteur pondérateur, l'indice de pollution sédimentaire, définit comme une fonction linéaire de la somme de facteurs d'enrichissement et introduit par Müller (1969) a été calculé (équation 3).

$\mathrm{IPS}=\frac{\sum\left(\mathrm{FE}_{\mathrm{m}} \times W_{\mathrm{m}}\right)}{\sum W_{\mathrm{m}}}$

- $W_{\mathrm{m}}$ : poids de toxicité ou facteur pondérateur du métal $\mathrm{m}$.

- $\mathrm{FE}_{\mathrm{m}}$ : facteur d'enrichissement du métal $\mathrm{m} ; 0 \leq \mathrm{IPS}<2$ Sédiment sain ; $2 \leq$ IPS $<5$ Sédiment faiblement pollué; $5 \leq$ IPS $<10$ Sédiment moyennement pollué; $10 \leq$ IPS $<20$ Sédiment très pollué; IPS $\geq 20$ Sédiment dangereux 


\subsubsection{Indice de charge Polluante}

L'indice global (l'indice de charge métallique (PLI : Pollution Load Index en Anglais)) d'étude de la contamination métallique des sédiments à partir de la concentration totale de tous les métaux étudiés et qui permet d'estimer le degré de contamination global des sédiments d'une zone étudiée a été utilisé. L'équation (4) suivante a été développée par Tomlinson et al. (1980), pour le calcul de l'indice de charge polluante (PLI).

$\mathrm{PLI}=\sqrt[n]{\mathrm{FC}_{1} \times \mathrm{FC}_{2} \times \mathrm{FC}_{3} \times \ldots \times \mathrm{FC}_{\mathrm{n}}}$

ou

$\mathrm{FC}_{i}=\frac{C_{D}^{i}}{C_{R}^{i}}$

$\mathrm{FC}_{i}$ : facteur de contamination du métal $i . \mathrm{PLI}=0$ pas de détérioration; $\mathrm{PLI}=1$ présents aux concentrations de référence; PLI > 1 détérioration progressive du milieu.

\subsubsection{Indice de risque écologique potentiel (RI) et risque potentiel de chaque ETM pris individuellement}

Cet indice est introduit pour évaluer le degré de risque écologique des éléments traces dans les sédiments. Les valeurs de RI se calculent à partir des formules de Häkanson (1980) suivantes (équation 5)

$\mathrm{RI}=\sum_{i}^{n} E_{r}^{i} ; E_{r}^{i}=T_{f}^{i} \times C_{f}^{i} ; C_{f}^{i}=\frac{C_{D}^{i}}{C_{R}^{i}}$ ou :

$C_{f}^{i}$ : coefficient de contamination; $C_{D}^{i}$ : concentration de l'ETM, $C_{R}^{i}$ : concentration de l'ETM dans les sédiments non pollués; Tif; facteur de toxicité de l'ETM. Les poids respectifs assigné aux métaux utilisés pour les calculs de IPS et RI sont : $\mathrm{Cd}=30 ; \mathrm{Hg}=40, \mathrm{As}=10 ; \mathrm{Pb}=5 ; \mathrm{Cu}=2$; $\mathrm{Zn}=1)$; $\mathrm{RI}>150$ risque bas : $150 \leq \mathrm{RI}<300$ risque modéré; $300 \leq \mathrm{RI}<600$ risque considérable; $\mathrm{RI} \geq 600$ risque très élevé.

\section{Résultats et Discussion}

\subsection{Variabilité saisonnière et étude comparative}

Les résultats des concentrations totales des ETMs dans les sédiments du lac Guessabo sont présentés dans le tableau 1.

Les concentrations moyennes des éléments traces métalliques lors de cette étude, comparées à celles des valeurs de pollution préindustrielle, mettent en évidence une contamination métallique des sédiments du lac.

Les valeurs des concentrations totales $\mathrm{Mn}$ et $\mathrm{Zn}$ (tableau 1) à toutes les stations sont inférieures aux valeurs préindustrielles, suggérant que les teneurs de ces éléments sont inférieures à ceux de la croute continentale. Cependant, les valeurs des concentrations totales de $\mathrm{Hg}, \mathrm{Pb}$, As et $\mathrm{Cu}$ (tableau
1) à toutes les stations sont supérieures aux valeurs préindustrielles excepté une station pour le plomb (G3) et l'arsenic (G4), ce qui suggère que ces stations sont enrichies par ces métaux. La forte croissance démographique et le développement économique ont contribué de manière significative à la détérioration actuelle de la qualité de l'eau, y compris l'accumulation de métaux lourds tels que le $\mathrm{Hg}, \mathrm{Cd}, \mathrm{Pb}, \mathrm{As}$ et $\mathrm{Cu}$ dans le milieu aquatique (Rajeshkumar et al., 2017). Les résultats obtenus dans les sédiments du lac Guessabo du présent travail n'ont pu être comparés avec ceux d'un travail antérieur mené dans le même lac en raison d'absence d'étude de ce type dans cette ressource. En revanche, les sédiments du lac Guessabo ont fait l'objet d'une autre comparaison avec ceux du fleuve Comoé du même pays. Nous avons noté différents degrés de contamination métallique par les éléments $\mathrm{Cu}, \mathrm{Zn}, \mathrm{Pb}$ et $\mathrm{Mn}$ dans les sédiments de ce fleuve en raison de la proximité de l'estuaire fleuve Comoé avec la plus agglomération qui est Abidjan (Keumean et al., 2013).

En ce qui concerne la variabilité saisonnière de ces paramètres (Fig. 2), l'analyse a permis de discriminer la saison sèche de la saison des pluies $(P<0,05)$. Durant la saison sèche, les teneurs en $\mathrm{Hg}$, en $\mathrm{Pb}$, en $\mathrm{Cd}$, en $\mathrm{Cu}$, en $\mathrm{Mn}$ et en Fe étaient importantes tandis qu'en saison des pluies, c'était l'As, qui présentait des valeurs élevées. Au plan spatial, les stations G2 et G5 présentaient généralement des teneurs élevées pour tous les éléments traces métalliques. Un ordre général de disponibilité des métaux lourds dans les sédiments a pu être établi : $\mathrm{Fe}>\mathrm{Cu}>\mathrm{Mn}>\mathrm{Pb}>\mathrm{As}>\mathrm{Hg}>\mathrm{Cd}$. Le faible taux d'accumulation des métaux (As et $\mathrm{Cd}$ ) dans les sédiments serait dû aux formes carbonates qui ont un rôle de diluant dans le développement des éléments de transition ( $\mathrm{Li}$ et al., 2018b). Les concentrations de $\mathrm{Pb}$ et de $\mathrm{Cu}$ étaient plus élevées dans les sédiments de surface par rapport aux $\mathrm{Cd}$, probablement en raison de la plus grande stabilité de leurs complexes (les oxydes de fer amorphes et les minéraux argileux) de surface ou sa faible abondance dans la nature. Les concentrations de métaux $(\mathrm{Hg}, \mathrm{Pb}, \mathrm{Cd}, \mathrm{Cu}, \mathrm{Mn}$ et $\mathrm{Fe})$ élevés dans les sédiments en saison sèche seraient dues au niveau bas de l'eau qui pourrait aider à accumuler les métaux lourds dans les sédiments (Mohammad Ali et al., 2016) ou aux apports d'eaux usées des sous- préfectures et villages en bordure du lac. De plus, la diminution de la concentration en saison pluvieuse pourrait être due aux précipitations, qui pourraient augmenter le processus de «lixiviation » et contribuer à la dilution des métaux lourds pendant la saison des pluies qui s'opèrent par des apports de sédiments moins ou non contaminés (Duman et Kar, 2012). 
Tableau 1. Teneur moyenne des éléments traces et valeurs Préindustrielles.

\begin{tabular}{|c|c|c|c|c|c|c|c|c|}
\hline $\begin{array}{l}\text { Métaux } \\
\text { louds }\end{array}$ & $\begin{array}{r}\text { Valeurs } \\
\text { préindustrielles }\end{array}$ & G1 & $\mathrm{G} 2$ & G3 & G4 & G5 & G6 & G7 \\
\hline $\mathrm{Hg}(\mu \mathrm{g} / \mathrm{g})$ & 0.056 & $0.47 \pm 0.24$ & $0.61 \pm 0.11$ & $0.68 \pm 0.55$ & $0.81 \pm 0.52$ & $0.41 \pm 0.06$ & $0.14 \pm 0.19$ & $0.35 \pm 0.19$ \\
\hline $\mathrm{Cd}(\mu \mathrm{g} / \mathrm{g})$ & 0.102 & $0.22 \pm 0.29$ & $0.26 \pm 0.18$ & $0.25 \pm 0.18$ & $0.03 \pm 0.02$ & $0.07 \pm 0.04$ & $0.04 \pm 0.05$ & $0.08 \pm 0.14$ \\
\hline $\mathrm{Pb}(\mu \mathrm{g} / \mathrm{g})$ & 17 & $47.91 \pm 30.46$ & $34.75 \pm 17.95$ & $14.88 \pm 9.35$ & $18.86 \pm 20.96$ & $30.20 \pm 14.16$ & $15.34 \pm 8.53$ & $11.28 \pm 7.76$ \\
\hline As $(\mu \mathrm{g} / \mathrm{g})$ & 2 & $2.53 \pm 1.71$ & $2.67 \pm 1.14$ & $2.10 \pm 0.77$ & $1.41 \pm 0.43$ & $3.46 \pm 0.36$ & $2.55 \pm 0.97$ & $3.09 \pm 0.61$ \\
\hline $\mathrm{Cu}(\mu \mathrm{g} / \mathrm{g})$ & 14.3 & $39.29 \pm 11.63$ & $45.49 \pm 15.80$ & $163.20 \pm 121.13$ & $157.63 \pm 137.59$ & $278.42 \pm 155.81$ & $230.25 \pm 195.55$ & $178.60 \pm 170.49$ \\
\hline $\operatorname{Mn}(\mu \mathrm{g} / \mathrm{g})$ & 527 & $21.34 \pm 2.85$ & $43.83 \pm 6.96$ & $19.62 \pm 8.73$ & $77.09 \pm 22.82$ & $28.61 \pm 4.56$ & $51.13 \pm 7.94$ & $63.06 \pm 13.62$ \\
\hline $\mathrm{Zn}(\mu \mathrm{g} / \mathrm{g})$ & 52 & $7.96 \pm 5.95$ & $45.89 \pm 27.74$ & $14.97 \pm 8.93$ & $5.77 \pm 5.27$ & $14.16 \pm 16.12$ & $9.11 \pm 3.72$ & $10.07 \pm 10.60$ \\
\hline $\mathrm{Fe}(\mathrm{g} / \mathrm{kg})$ & 30.890 & $19.36 \pm 11.31$ & $33.81 \pm 13.97$ & $21.65 \pm 10.96$ & $32.86 \pm 9.92$ & $35.52 \pm 19.35$ & $30.09 \pm 6.51$ & $15.71 \pm 5.39$ \\
\hline
\end{tabular}

\subsection{Diagnostic des contaminations sédimentaires}

\subsubsection{Facteurs d'enrichissement (FE) et indices de pollution sédimentaires (IPS)}

Le tableau 2 montre les valeurs moyennes du facteur d'enrichissement (FE) et de l'indice de pollution sédimentaire des éléments traces dans les sédiments des stations du lac Guessabo. Les résultats montrent une absence d'enrichissement des sédiments en Mn et en Zn à toutes les stations par ces métaux $(\mathrm{FE}<1)$ dans le lac. Ils montrent que ces éléments sont principalement d'origine terrigène (Diop et al., 2015). Pour le $\mathrm{Pb}$ et le $\mathrm{Cd}$ l'enrichissement varie d'aucun enrichissement à enrichissement modéré pour les sédiments $(1>\mathrm{FE}<5)$. Toutes les stations présentent un enrichissement modéré à un enrichissement sévère pour le $\mathrm{Hg}(3<\mathrm{FE}<20)$. Pour ce qui est du $\mathrm{Cu}$, l'on a observé un enrichissement mineur à modérément sévère $(1<\mathrm{FE}<20)$ au niveau des sédiments. Toutes les stations présentent un enrichissement mineur à modérément sévère pour l'As $(1>\mathrm{FE}<10)$ au niveau des sédiments. Les stations G1, G3, G4 et G7 présentent les valeurs de facteurs d'enrichissement, les plus élevées (modérément sévères) pour le mercure indiquant qu'ils sont fortement enrichis et que l'origine de cet enrichissement est principalement anthropique (Diop et al., 2015). L'origine anthropique du cuivre aux stations G5, G6 et G7 serait dû aux additifs nutritionnels contenant du cuivre utilisés pour l'engraissement des porcs ou/et aux fertilisants dans l'agriculture. Pour l'évaluation de la qualité globale des sédiments, tenant en compte d'une approche multi-métaux, incluant de la toxicité relative de chaque métal, l'indice de pollution sédimentaire (IPS) a été calculé (tableau 2). Selon la classification de Singh et al. (2002), les sédiments ont été trouvés faiblement pollués $(2<$ IPS $<5)$ dans les stations G5 et G6, modérément pollués $(5<$ SPI $<10)$ aux autres stations. Dans le lac, les valeurs de IPS par points de prélèvement décroissent en suivant l'ordre : G3 $>$ G1 $>$ G7 $>$ G4 $>$ G2 $>$ G5 $>$ G6. Ce classement s'explique aisément par les caractéristiques des effluents : les sédiments des stations G1, G3 et G7 sont directement impactés par les eaux usées non traitées et le ruissellement agricole (engrais et pesticides).
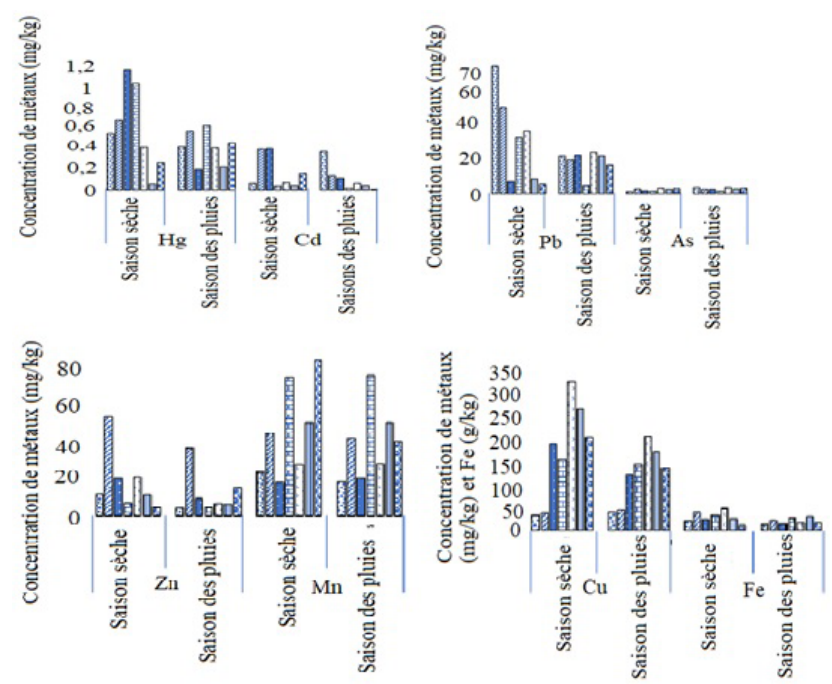

Figure 2. Variations des concentrations des éléments traces métalliques dans les sédiments du lac Guessabo.

\subsubsection{Indice de géoaccumulation}

Le tableau 3 indique les valeurs de l'indice de géoaccumulation des différents points d'échantillonnages et pour chaque métal. Les index de géoaccumulation du $\mathrm{Fe}$, du Mn et du $\mathrm{Zn}$ sont tous $<0$ à toutes les stations montrant que les sédiments du lac Guessabo ne sont pas pollués par ces ETMs. Les classes Igeo de $\mathrm{Pb}$ étaient respectivement " Modérément contaminé " et "Non contaminé à modérément contaminé " pour les sédiments des stations G7 et autres (G1, G2, G5), tandis que la classe Igeo de As et Cd était “ Non contaminé à modérément contaminé " pour les sédiments des stations G1, G2, G3 pour Cd et G5, G7 pour As selon la classification de Müller (1981). Les classes Igeo de Hg étaient respectivement «Le Sévèrement contaminées » et « Modérément contaminées à sévèrement contaminées » pour les sédiments des stations G4 et autres (G1, G2, G3, G5, G7), tandis que les classes Igeo de $\mathrm{Cu}$ étaient respectivement «Sévèrement contaminées » et « Modérément contaminée à sévèrement contaminée » pour les sédiments des stations G5, G6, G7 et autres (G2, G3, G4) selon la classification de Muller (1981). 
Tableau 2. Récapitulatif des valeurs moyennes du facteur d'enrichissement et de l'indice de pollution sédimentaire par station et par élément métallique dans les sédiments du lac Guessabo.

\begin{tabular}{llrrrrrrrr}
\hline Millieu d'étude & Stations & \multicolumn{9}{c}{ FE } & \multirow{3}{*}{ IPS } \\
\cline { 3 - 8 } & & $\mathrm{Mn}$ & $\mathrm{Cu}$ & $\mathrm{Zn}$ & $\mathrm{Pb}$ & $\mathrm{As}$ & $\mathrm{Cd}$ & $\mathrm{Hg}$ & \\
\hline Guessabo Lake & $\mathrm{G} 1$ & 0.06 & 2.52 & 0.17 & 4.49 & 2.02 & 3.49 & 13.50 & 7.74 \\
& $\mathrm{G} 2$ & 0.07 & 1.67 & 0.58 & 1.86 & 1.22 & 2.42 & 9.91 & 5.53 \\
& $\mathrm{G} 3$ & 0.05 & 9.36 & 0.30 & 1.25 & 1.50 & 3.63 & 17.00 & 9.37 \\
& $\mathrm{G} 4$ & 0.12 & 5.95 & 0.07 & 1.04 & 0.66 & 0.28 & 13.59 & 6.50 \\
& G5 & 0.04 & 9.74 & 0.17 & 1.54 & 1.50 & 0.61 & 6.31 & 3.64 \\
& G6 & 0.09 & 9.50 & 0.13 & 0.92 & 1.30 & 0.48 & 2.56 & 1.84 \\
& G7 & 0.21 & 14.13 & 0.27 & 1.30 & 3.03 & 1.67 & 12.37 & 7.02 \\
& Moyenne & 0.09 & 7.55 & 0.24 & 1.77 & 1.60 & 1.79 & 10.75 & 5.95 \\
\hline
\end{tabular}

Tableau 3. Récapitulatif des indices de géoaccumulation des éléments métalliques dans les sédiments du lac Guessabo.

\begin{tabular}{llrrrrrrrr}
\hline Millieu & & \multicolumn{7}{c}{ Indice de géoaccumulation } \\
\cline { 3 - 10 } d'étude & Stations & $\mathrm{Fe}$ & $\mathrm{Mn}$ & $\mathrm{Cu}$ & $\mathrm{Zn}$ & $\mathrm{Pb}$ & $\mathrm{As}$ & $\mathrm{Cd}$ & $\mathrm{Hg}$ \\
\hline Guessabo Lake & $\mathrm{G} 1$ & -1.26 & -5.21 & 0.87 & -3.29 & 0.91 & -0.24 & 0.51 & 2.49 \\
& $\mathrm{G} 2$ & -0.45 & -4.17 & 1.08 & -0.76 & 0.45 & -0.16 & 0.79 & 2.85 \\
& $\mathrm{G} 3$ & -1.09 & -5.33 & 2.92 & -2.38 & -0.77 & -0.51 & 0.73 & 2.99 \\
& $\mathrm{G} 4$ & -0.49 & -3.35 & 2.87 & -3.75 & -0.43 & -1.08 & -2.35 & 3.26 \\
& $\mathrm{G} 5$ & -0.38 & -4.78 & 3.69 & -2.46 & 0.24 & 0.20 & -1.13 & 2.27 \\
& $\mathrm{G} 6$ & -0.62 & -3.95 & 3.42 & -3.09 & -0.73 & -0.23 & -1.76 & 0.74 \\
& $\mathrm{G} 7$ & -1.56 & -3.68 & 3.05 & -2.48 & 1.17 & 0.04 & -0.84 & 2.07 \\
& Moyenne & -0.84 & -4.35 & 2.56 & -2.60 & -0.22 & -0.28 & -0.58 & 2.38 \\
\hline
\end{tabular}

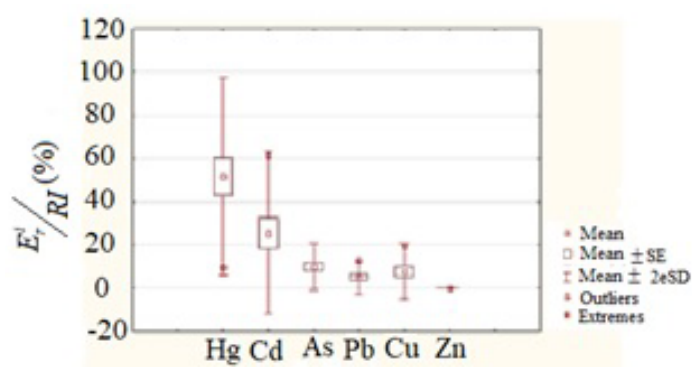

Figure 3. Contribution des métaux respective au RI.

Ces résultats concordent assez bien avec ceux obtenus par l'évaluation au Facteur d'enrichissement.

\subsubsection{Indice de charge polluante (PLI)}

Le tableau 4 montre les valeurs de l'indice de charge polluante. Les stations G2, G3 et G5 ont des valeurs PLI supérieures à 1 , indiquant une détérioration progressive du lac en ces stations. La raison probable de cette contamination serait les déchets agricoles (engrais et pesticides) et les eaux usées ainsi que les déchets des exploitations piscicoles trouvées dans la partie où se trouve la station G5 (Men et al., 2018).
Tableau 4. Valeurs moyennes du PLI des sédiments par station.

\begin{tabular}{llr}
\hline Millieu d'étude & Stations & PLI \\
\hline Guessabo Lake & G1 & 0.96 \\
& G2 & 1.45 \\
& G3 & 1.1 \\
& G4 & 0.94 \\
& G5 & 1.23 \\
& G6 & 0.87 \\
& G7 & 0.97 \\
& Moyenne & 1.08 \\
\hline
\end{tabular}

3.2.4 Indices de risque écologique individuel $\left(\mathrm{E}_{r}^{i}\right)$ et de risque écologique potentiel (RI)

Le tableau 5 montre les valeurs des indices de risque écologique individuel et de risque écologique potentiel (RI) par station.

Le risque écologique moyen lié aux $\mathrm{Cd}, \mathrm{As}, \mathrm{Pb}, \mathrm{Cu}$ et $\mathrm{Zn}$ est largement inférieur à 40 traduisant un risque bas en référence à ces métaux. En revanche, celui lié au mercure est de 86,6 (> 40), montrant un risque considérable. Sur l'ensemble des échantillons de sédiments du lac Guessabo analysés, le résultat révèle un indice du risque écologique potentiel égale à 153,19 indiquant une pollution modérée (tableau 5). 
Tableau 5. Récapitulatif des Indices du risque écologique individuel $\left(E_{\mathrm{r}}^{\mathrm{i}}\right)$ et du risque écologique potentiel (RI).

\begin{tabular}{|c|c|c|c|c|c|c|c|}
\hline \multirow[b]{2}{*}{ Stations } & \multicolumn{6}{|c|}{ Indices du risque écologique individuel $\left(E_{\mathrm{r}}^{\mathrm{i}}\right)$} & \multirow{2}{*}{$\begin{array}{l}\text { Risque écologique } \\
\text { potentiel (RI) }\end{array}$} \\
\hline & $\mathrm{Hg}$ & $\mathrm{Cd}$ & As & $\mathrm{Pb}$ & $\mathrm{Cu}$ & $\mathrm{Zn}$ & \\
\hline G1 & 9.48 & 59.66 & 12.67 & 12.61 & 2.38 & 0.08 & 97 \\
\hline G2 & 121.5 & 72.27 & 13.38 & 9.15 & 2.75 & 0.48 & 219 \\
\hline G3 & 133.5 & 69.47 & 10.52 & 3.95 & 9.89 & 0.15 & 227 \\
\hline G4 & 162 & 8.18 & 7.06 & 4.96 & 9.55 & 0.06 & 191 \\
\hline G5 & 81.3 & 19.09 & 17.30 & 7.94 & 16.87 & 0.14 & 143 \\
\hline G6 & 28 & 12.27 & 12.75 & 4.04 & 13.95 & 0.09 & 71 \\
\hline G7 & 70.5 & 23.18 & 15.45 & 2.97 & 10.82 & 0.1 & 123 \\
\hline
\end{tabular}

Les résultats de l'indice de risque écologique individuel indiquent que les concentrations de $\mathrm{Hg}$ sont susceptibles d'avoir des effets nocifs sur les organismes vivant dans les sédiments. $\mathrm{Hg}$ contribue en moyenne à $51,7 \%$ au risque écologique potentiel suivit du Cd qui contribue à $25,57 \%$ (Fig. 3). Ces métaux lourds sont susceptibles d'affecter négativement une diversité de communauté microbienne (Huang et al., 2016). Les concentrations élevées de mercure et de cuivre dans les sédiments du lac Guessabo constituent donc un danger potentiel et font de ces sédiments une source endogène de pollution. En effet, ces polluants métalliques peuvent passer dans la colonne d'eau lorsque les conditions sont favorables, engendrant ainsi des effets néfastes sur la qualité des eaux et sur la vie aquatique. L'enrichissement en $\mathrm{Hg}$ enregistré dans cette zone serait sans doute dû aux principales sources anthropogéniques qui sont l'agriculture (insecticides, fongicides, bactéricides et herbicides), la peinture, les appareils électriques et les produits pharmaceutiques. La mauvaise gestion des déchets dans la zone serait à l'origine de cette pollution. L'As, le $\mathrm{Cd}$, le $\mathrm{Pb}$ et $\mathrm{Hg}$ sont des éléments non essentiels qui ont des effets toxiques avérés sur les humains, en particulier les enfants et les adolescents (Whitehead et Buchanan, 2019). Ces effets englobent les maladies immunitaires, la défaillance d'un organe interne, les lésions neurologiques et les maladies respiratoires (Chikkanna et al., 2019).

\section{Conclusions}

Le présent travail avait pour objectif principal de connaître l'état de contamination des sédiments par les éléments traces métalliques et leur toxicité, il constitue un premier état des lieux.

Les résultats de Igeo et $\mathrm{FE}$ calculés, ont montré que les sédiments devaient être considérées comme modérément contaminées à sévèrement contaminées en $\mathrm{Hg}$, modérément contaminées en $\mathrm{Cu}$ et presque non contaminées par les autres éléments. Au regard de la pollution cumulative (PLI et IPS) et des risques écologiques potentiels, les sédiments du lac Guessabo se sont avérés être pollués par le $\mathrm{Hg}$, qui a contri- bué à 51,7\% des risques écologiques potentiels et dans une moins mesure par $\mathrm{Cd}$, As et le $\mathrm{Pb}$.

Collaborateurs. KSK, corresponding author, he wrote the manuscript. KBK contributed in samplings and processing of data. KFK assisted in proofreading the manuscript KCB participated in the interpretation of the results KMK participated in sampling campaigns and data analysis KOUAKOU Kouassi Lazare helped in the making of maps.

Intérêts concurrents. Les auteurs déclarent qu'ils n'ont aucun conflit d'intérêts.

Clause de non-responsabilité. Publisher's note : Copernicus Publications remains neutral with regard to jurisdictional claims in published maps and institutional affiliations.

Déclaration du numéro spécial. This article is part of the special issue "Hydrology of Large River Basins of Africa". It is a result of the 4th International Conference on the "Hydrology of the Great Rivers of Africa", Cotonou, Benin, 13-20 November 2021.

Remerciements. Nous adressons nos sincères remerciements au directeur du Laboratoire National d'Appui au Développement Agricole. Cette institution nous a fourni un cadre et des facilités de travail remarquable pour l' analyse des échantillons. Les auteurs remercient les évaluateurs du Bulletin pour leurs corrections et commentaires.

\section{Références}

Beutel, M. W., Leonard, T. M., Dent, S. R., and Moor, B. C. : Effects of aerobic and anaerobic conditions on $\mathrm{P}, \mathrm{N}, \mathrm{Fe}, \mathrm{Mn}$ and $\mathrm{Hg}$ accumulation in waters overlaying profundal sediments of an oligo-mesotrophic lake, Water Res., 42, 1953-1962, 2008.

Chikkanna, A., Mehan, L., Sarath, P. K., and Ghosh, D. : Arsenic Exposures, Poisoning, and Threat to Human Health : Arsenic Af- 
fecting Human Health. In Environmental Exposures and Human Health Challenges, IGI Global, 86-105, 2019.

Daskalakis, K. D. and O'Connor, T. P. : Normalization and elemental sediment contamination in the Coastal United States, Environ. Sci. Technol., 29, 470-477, 1995.

Diop, C., Dewaelé, D., Cazier, F., Diouf, A., and Ouddane, B. : Assessment of trace metals contamination level, bioavailability and toxicity in sediments from Dakar coast and Saint Louis estuary in Senegal, West Africa, Chemosphere, 138, 980-987, https://doi.org/10.1016/j.chemosphere.2014.12.041, 2015.

Duman, F. and Kar, M. : Temporal variation of metals in water, sediment and tissues of the European Chup (Squalius cephalus L.), Bull. Environ. Contam. Toxicol, 89, 428-433, 2012.

Häkanson, L. : An ecological risk index for aquatic pollution control. A sedimentological Approach, Water Res., 14, 9751001, 1980.

Huang, Y., Zhang, D., Xu, Z., Yuan, S., Li, Y., and Wang, L. : Effect of overlying water $\mathrm{pH}$, dissolved oxygen and temperature on heavy metal release from river sediments under laboratory conditions, Archives of Environmental Protection, 43, 28-36, 2017.

Keumean, K. N., Bamba, S. B., Soro, G., Soro, N., Metongo, S. B., et Biemi, J. : Concentration en métaux lourds des sédiments de l'estuaire du fleuve Comoé à Grand-Bassam (Sud-Est de la Côte d'Ivoire), J. Appl. Biosci, 61, 4530-4539, 2013.

Kouamé, K. B., Konan, K. S., Attoungbre, K. S., Konan, K. F., Boussou, K. C., and Kouamé, K. M. : Physicochemical and Bacteriological Parameters of Surface Water Quality in part of West Côte d'Ivoire : Potential Resources for Drinking Water Production (Guessabo Lake), J. Appl. Environ. Biol. Sci., 9, 17-29, 2019a.

Kouame, K. B., Konan, K. S., Konan, K. F., Boussou, K. C., and Kouassi, K. L. : Heavy metals contamination of an aquatic environment and health risks assessment in two fish species : case of the Guessabo lake, western Côte d'Ivoire, Orient. J. Chem., 35, 1742-1755, 2019b.

Li, Q., Na, G., Zhang, L., Lu, Z., Gao, H., and Li, R. : Effects of corresponding and non-corresponding contaminants on the fate of sulfonamide and quinolone resistance genes in the Laizhou Bay, China., Mar. Pollut. Bull., 128, 475-482, 2018b.
Malferrari, D., Brigatti, M. F., Laurora, A., and Pini, S. Heavy metals in sediments from canals for water supplying and drainage : mobilization and control strategies, J. Hazard. Mater. 161, 723729, 2009.

Men, C., Liu, R. M., Xu, F., Wang, Q. R., Guo, L. J., and Shen, Z. Y. : Pollution characteristics, risk assessment, and source apportionment of heavy metals in road dust in Beijing, China., Sci. Total Environ., 612, 138-147, https://doi.org/10.1016/j.scitotenv.2017.08.123, 2018.

Mohammad, A. M., Mohammad, L. A., Saiful, I. M., and Zillur, R. M. : Preliminary assessment of heavy metals in water and sediment of Karnaphuli River, Bangladesh, Environ. Nanotec. Monit. Manage. 5, 27-35, 2016.

Müller, G. : Die Schwemetallbelastung der sedimente des Neckars und seiner Nebenflusse : eine Bestandsanfnahme, Chem. Zeitung, 105, 157-164, 1981.

Müller, G. : Index of geoaccumulation in sediments of the Rhine River, Geojournal, 2, 109-118, 1969.

Olivares-Rieumont, S., de la Rosa, D., Lima, L., Graham, D. W., Alessandro, K. D., Borroto, J., Martınez, F., and Sanchez, J. : Assessment of heavy metal levels in Almendares River sedimentsHavana City, Cuba, Water Res., 39, 3945-3953, 2005.

Rajeshkumar, S., Liu, Y., Zhang, X., Ravikumar, B., Bai, G., and Li, X. : Studies on seasonal pollution of heavy metals in water, sediment, fish and oyster from the Meiliang Bay of Taihu Lake in China, Chemosphere, 191, 626-638, 2017.

Wang, C., Liu, S., Zhao, Q., Deng, L., and Dong, S. : Spatial variation and contamination assessment of heavy metals in sediments in the Manwan Reservoir, Lancang River., Ecotoxicology and Environmental Safety, 82, 32-39, 2012.

Whitehead, L. T. S. and Buchanan, S. D. : Childhood Lead Poisoning : A Perpetual Environmental Justice Issue, J. Public Health Manag Pract., 1 115-120, 2019. 